\title{
Online Reports of Foodborne Illness Capture Foods Implicated in Official Foodborne Outbreak Reports
}

\author{
Elaine O. Nsoesie ${ }^{\star 1,2}$, Sheryl A. Kluberg ${ }^{1}$ and John S. Brownstein ${ }^{1,2}$ \\ 'Boston Children's Hospital, Boston, MA, USA; ${ }^{2}$ Harvard Medical School, Boston, MA, USA
}

\section{Objective}

We assessed whether foodservice reviews on Yelp.com (a business review site) can be used to support foodborne illness surveillance efforts.

\section{Introduction}

Traditional surveillance systems only capture a fraction of the estimated 48 million yearly cases of foodborne illness in the United States due to few affected individuals seeking medical care and lack of reporting to appropriate authorities. Non-traditional disease surveillance approaches could be used to supplement foodborne illness surveillance systems.

\section{Methods}

We obtained reviews from 2005-2012 of 5824 foodservice businesses closest to 29 colleges. After extracting recent reviews describing episodes of foodborne illness, we compared implicated foods to foods in outbreak reports from the U.S. Centers for Disease Control and Prevention (CDC).

\section{Results}

Broadly, the distribution of implicated foods across five categories was as follows: aquatic (16\% Yelp, $12 \%$ CDC), dairy-eggs $(23 \%$ Yelp, 23\% CDC), fruits-nuts (7\% Yelp, 7\% CDC), meat-poultry (32\% Yelp, 33\% CDC), and vegetables (22\% Yelp, 25\% CDC). The distribution of foods across 19 more specific food categories was also similar, with spearman correlations ranging from 0.60 to 0.85 for 2006-2011. The most implicated food categories in both Yelp and CDC were beef, dairy, grains-beans, poultry and vine-stalk.

\section{Conclusions}

Based on observations in this study and the increased usage of social media, we posit that online illness reports could complement traditional surveillance systems by providing near real-time information on foodborne illnesses, implicated foods and locations.

\section{Keywords}

foodborne illness; foodborne diseases; disease surveillance; social media; gastroenteritis

\section{*Elaine O. Nsoesie}

E-mail: elaine.nsoesie@childrens.harvard.edu 